\title{
Mouse sequencing plan aims to boost models
}

Helen Pearson, New York

Efforts to find genes involved in human disease are set to benefit from a plan to sequence the genetic code of 15 different mouse strains.

Lab mice might look alike, but in fact scientists work with at least 100 different inbred strains. These strains differ hugely in their behaviour, physiology and susceptibility to disease, and researchers are keen to find the differences in the genetic sequences that underlie these traits, with the aim of locating counterparts in humans.

The sequencing project plans to decipher the genomes of 15 mouse strains within two years. It will be largely funded by the US National Institute of Environmental Health Sciences and the sequencing will be done by Perlegen Sciences, based in Mountain View, California. "People are delighted," says mouse geneticist Kenneth Paigen of the Jackson Laboratory in Bar Harbor, Maine.

Researchers have long puzzled over the differences between mouse strains (see Nature 415, 8-9; 2002). After a few weeks on a high-fat diet, for example, one strain's cholesterol level hovers at a healthy 100 milligrams per decilitre, whereas another's rockets to more than five times that amount.

Tracking down the genes involved in conditions such as high cholesterol, diabetes, obesity or cancer is a long process. Researchers cross a mouse strain susceptible to diabetes, for example, with a diseaseresistant strain, and identify the chunks of the genome that are inherited with the disease. They then laboriously scour the DNA in these regions for 'susceptibility genes'.

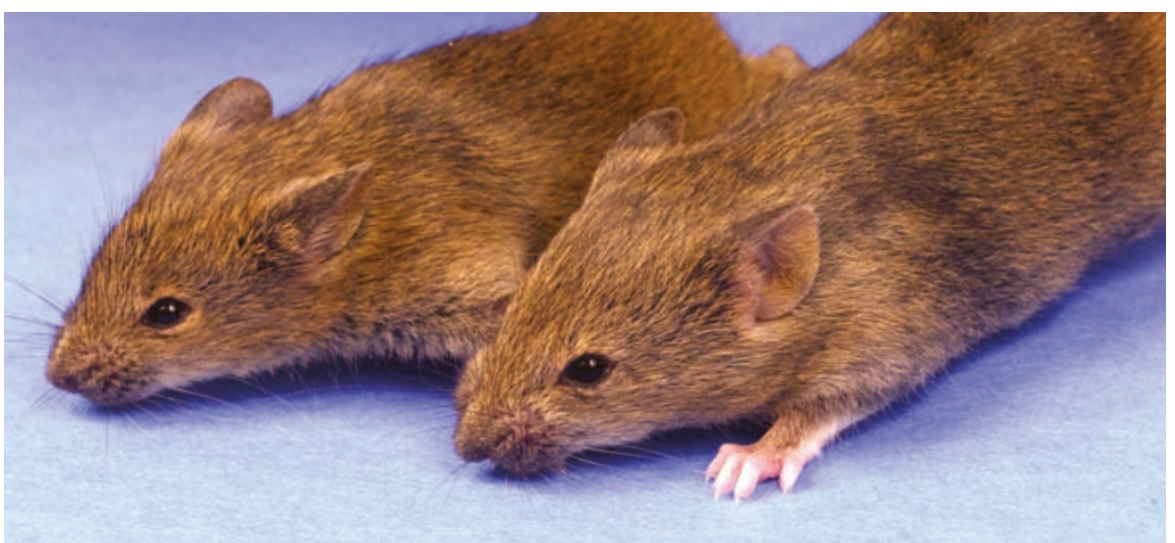

Take the strain: a catalogue of genetic differences between mouse breeds will aid disease research.

The new line-up of mouse genomes should accelerate the process significantly. The genomic data will show researchers exactly how the DNA of the diabetes-prone strain differs from that of other strains. So researchers should be able to home in quickly on relevant genes and perhaps find corresponding ones involved in human disease.

Geneticists have had access to the genome of one widely used mouse strain, called C57BL/6J, since 2002 - and, for a fee, genomes of a few other strains are available from Celera Genomics in Rockville, Maryland. The project's organizers say this is the most comprehensive attempt yet to analyse the genomes of different strains, and that the code will be deposited in public databases.

The 15 strains were selected because they are widely used in research, are evolutionarily diverse and show different propensities to common diseases.

Researchers hope to link their genetic information to the Mouse Phenome Project, an international effort launched in 2000 to collate the anatomical, physiological and behavioural differences between mouse strains. This should help researchers to choose the most appropriate strain for studying a disease.

Ultimately, the amount of detailed biological and genetic data collected might be so great that it eliminates the need for many mouse crosses altogether, says the leader of the Phenome Project, Molly Bogue, also at the Jackson Laboratory. Researchers could simply select a panel of mouse strains with different cholesterol levels, and use computer analyses to help pinpoint the candidate genes that differ between them. "That's what we're hoping for," she says.

\section{Green groups baulk at joining nanotechnology talks}

\section{Kendall Powell}

One of the first efforts to get parties round the table to discuss nanotechnology has got off to a faltering start, after leading environmental groups declined to take part.

The International Council on

Nanotechnology (ICON) has been set up to drive open discussion about the benefits and pitfalls of the field, which comprises a clutch of technologies involving materials and components on the scale of a billionth of a metre. The council held its first meeting on 28 October at Rice University in Houston, Texas, which has strong research programmes in the implications of nanotechnology.

But the three main environmental groups invited to participate said they were not ready to do so, complaining that the council was likely to be biased because it depended on industry funds.

Jennifer Sass of the National Resources
Defense Council and Scott Walsh of Environmental Defense, both based in Washington DC, and Pat Mooney of the Canadian ETC Group, based in Ottawa, all turned down invitations to join ICON. Walsh and Mooney participated in last week's meeting as guests.

Researchers and industrial backers of nanotechnology hope that discussion of its effects will help avoid the public mistrust that has plagued fields such as agricultural biotechnology.

"We welcome anyone who thinks they have a stake in this discussion," says Kristen Kulinowski, director for education and public policy at Rice's Center for Biological and Environmental Nanotechnology, which manages ICON. Kulinowski says the council discussed how to make its decision-making truly independent of its funding sources.

Sass would prefer the council to be publicly funded. Mooney says he would join if ICON included more members from the academic world, from trade unions and from developing countries.

But academic members say the current arrangements are satisfactory. "I'm not concerned about industry sponsorship," says Günter Oberdörster, a toxicologist at the University of Rochester in New York, who studies nanoparticles. "I am concerned about non-governmental organizations possibly not being part of it," he says. "We should find the answer together, in a transparent process."

William Provine, a council member who tracks nanotechnology applications for DuPont, the chemicals company based in Wilmington, Delaware, agrees. "We need to establish a credible group of stakeholders," he says, "and we have not gotten there, yet." 